\title{
ICT4Depression: service oriented architecture applied to the treatment of depression
}

\author{
Artur Rocha and Mario Ricardo Henriques \\ INESC TEC (formerly INESC Porto) \\ Porto, Portugal \\ \{artur.rocha, ricardo.henriques\}@inescporto.pt \\ Michel Klein and Gabriele Modena \\ Faculty of Sciences, Vrije Universiteit \\ Amsterdam, The Netherlands \\ michel.klein@cs.vu.nl,g.modena@vu.nl \\ Eric Tousset and Thibaut Gauthier \\ AARDEX \\ Liege, Belgium \\ \{eric.tousset, thibaut.gauthier\}@ aardexgroup.com
}

\author{
João Correia Lopes and Rui Camacho \\ INESC TEC (formerly INESC Porto) and FEUP \\ Porto, Portugal \\ \{jlopes, rcamacho\}@fe.up.pt
}

Pepijn Van de Ven and Elaine McGovern

University of Limerick, Ireland

\{pepijn.vandeven, elaine.mcgovern\}@ul.ie

Lisanne Warmerdam

Faculty of Psychology, Vrije Universiteit

Amsterdam, The Netherlands

e.h.warmerdam@vu.nl

\begin{abstract}
FP7 ICT4Depression project aims at providing a set of tools to further improve both patient outcome and increase of access to treatment of the patients suffering from major depression.

This article describes the Information Systems (IS) architecture used in the project. ICT4Depression uses a service oriented architecture as means of bringing together different kinds of information concerning the patient, the therapeutic modules he is advised to follow and the sensors used to assess his status.
\end{abstract}

\section{Introduction}

Major depression is currently the fourth disorder worldwide in terms of disease burden, and is expected to be the disorder with the highest disease burden in high-income countries by 2030 . Current treatment methods can reduce the burden of this disease by approximately one third [11]. Although several effective treatments are available for depression in primary care, including pharmacotherapy and psychotherapy [9], there is much room for improvement of outcomes of these treatments. In FP7 ICT4Depression project, we developed a system aimed at improving the outcome of treatments for depression, featuring several innovative aspects to the clinical treatment of depression.
From the clinical perspective, this system builds up to a stepped care framework which makes use of ecological momentary assessment and intervention techniques (EMA and EMI) including the assessment of physiological symptoms in an integrated manner which enables timely interactive feedback and treatment adaptation if needed for patients and professionals alike [10]. From the software engineering perspective it maps to a service oriented architecture (SOA) constituting a federation of resources which client applications use and bind together to provide a tailored outcome.

In ICT4Depression, the SOA resources are made available by means of secure web services providing access to the underlying domain model for several psychotherapies (the interventions), to generate advanced feedback towards the best treatment course as well as providing tools for relapse prevention.

ICT4Depression also leverages on the popularity of smartphones, on the advances in the fields of mobile and pervasive computing and body area networks to provide an innovative client application for the Android platform. The mobile application not only provides access to the implemented psychotherapy interventions, but also alternative ways of assessment based on body-worn sensors.

Besides different forms of psychotherapy, the use of antidepressant medicines has also been shown to be effective in the treatment of depression. The effectiveness of prescribed antidepressant medication depends upon the adherence to dosing instructions [12], [14]. Correct dosing is 
necessary for the prescribed medication to work most effectively.

Psychotherapies and Pharmacotherapies offer two important options for the effective treatment of depression. Furthermore, it has also been showed that patients may benefit from their combination [8], [7], reason why the ICT4depression system addresses support for the selfmanagement of both.

\section{Requirements}

The corner stone of the system we present are the five therapeutic modules: psycho education and motivation, behavioral activation, problem solving therapy, cognitive restructuring and exercise therapy. They constitute the base of the self-help intervention, which ICT4Depression aims to bring even closer to the patient using the mobile phone in the role of a personal health assistant. Information enclosed in the exercises comprised in these therapies can also be complemented by using a web client.

Patient progress is measured by means of a web questionnaire and complemented by body-worn sensor information conveyed to the system by means of a smartphone. Some of the implemented psychotherapies take advantage of sensor information, like the Exercise Therapy, which can benefit from using mobility and activity information.

Another important input to monitor patient's progress are mood ratings, also collected by the mobile phone when the reasoning engine deems appropriate.

The reasoning engine is also in charge of providing the patient with personalized feedback and advising best treatment course.

The ICT4Depression system contains a sub-system dedicated to the monitoring of adherence. A good level of adherence is necessary for the prescribed medication to work most effectively. Information about patient's adherence may also be useful to the healthcare providers to take decision about the drug therapy. This sub-system supports the remote acquisition of adherence data, their transfer to a dedicated server and provides feedbacks to patients and healthcare providers.

In spite of the distributed and scalable characteristics of the system, particular attention has been given to the security of patient data.

Based on results of previous controlled trials, ICT4Depression will also undertake the opportunity to research about the use of sensors to detect acute emotional stress situations from the recording of heart rate and electrodermal activity.

In parallel with the use of the reasoning module as a tool for relapse prevention, we will also research about using inductive logic programming (ILP) as tool to attempt to predict relapses.

\section{Proposed Approach}

In order to respond to requirements (Section 2) a collection of software modules and sub-systems has been developed and deployed, binding together in the form of a service oriented architecture. The following sections describe these components.

\subsection{Overview of the ICT4Depression Sys- tem}

From a high-level perspective, the ICT4Depression system can be seen as a collection of self-management tools both for psychotherapies and pharmacotherapies, which take input from several monitoring sub-systems in order to provide timely assessment and supervision of the patient's treatment.

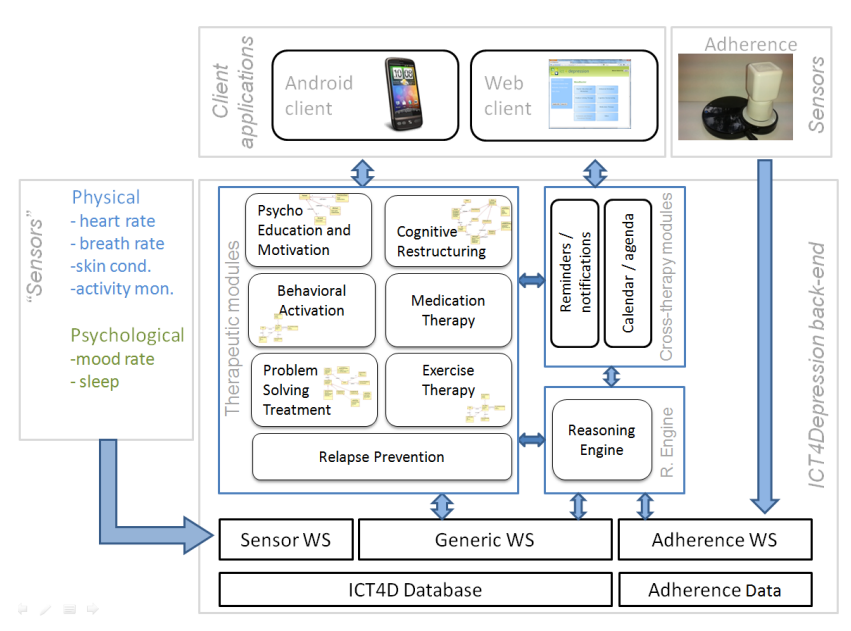

Figure 1. Overview of the ICT4Depression.

\subsection{Therapeutic modules}

The proposed approach consists of a set of modules accessible via website and smartphone (Android) by means of secure web services. These therapies are integrated in an intelligent self-help system leveraging on a Reasoning Engine (Section 3.4) and have been designed in close collaboration with the psychologist partners having in mind the end-user experience. All patients start with the Psycho-Education and Motivation module (PEM).

The PEM module helps the patient to assess the severity of problems and consists of general information about depression, goal setting, daily registration of mood and feedback about the level of mood during one week. It will also be used to give the patient advise about whether to start a treatment module, and which one. 
Behavioral Activation module aims to treat depression through techniques designed to reinforce pleasant and reduce unpleasant activities. The patient uses the Calendar/Agenda to schedule both activities and is advised to increase, if necessary to balance, the number of pleasant activities. It helps the patient during the day to work through the behavioral treatment by reminding of activities, prompting for mood ratings and giving personalized feedback.

The Problem Solving module aim to help the patient to regain control of his life by determining things that are important and how to cope with situations he cannot change. There is also defined in the module an iterative workflow to work around those problems that patient thought to be solvable.

The Cognitive Restructuring module helps the patient to work and control negative automatic thoughts, which are close connected with depression. This module will challenge the patient to reason if these are just interpretations and not actual truths. It aims to make the patient more logical about his beliefs and to consider all the evidences and be aware of the so-called thinking errors.

Exercise Therapy module aims to help patient to rise is exercise threshold, which is not only healthy and also a non-stigmatizing way of treatment depression. It his supported by the mobile phone mobility sensor in conjunction with Calendar/Agenda, to detect if the scheduled exercises/games have been performed and encourage the patient to increase his level of activity.

Medication Therapy aims providing adequate and personalized support for the management of patient drug intakes (see Section 3.3).

\subsubsection{Cross-therapy components}

One of the main goals of the project is assessing the progress in the patient's recovery. The Reasoning Engine (Section 3.4) collects data from the Therapeutic Modules and provides automatic feedback. Patients have then a better insight in the progress of their therapy, thus allowing them to increase its effectiveness.

The Calendar/Agenda works as a backbone interconnecting several Therapeutic Modules, by allowing the user to schedule activities, plan their exercises and helping the Reasoning Engine to determine the adequate timing for feedback reminders, notifications or rewards.

\subsection{Medical Adherence Sub-system}

\subsubsection{Architectural View}

As shown in Figure 2, the medication adherence sub-system is based on several components:

- the MEMS ${ }^{\circledR}$ monitor, a standalone device, records times of opening of the drug container. It also provides a direct visual feedback to the patient.

- the wireless reader downloads the data from the MEMS ${ }^{\circledR}$ monitor and transfers these data to a dedicated server.

- the adherence back-end stores adherence data, but also supports the interpretation of these data

- Web services provide secured access to information on adherence to various clients.

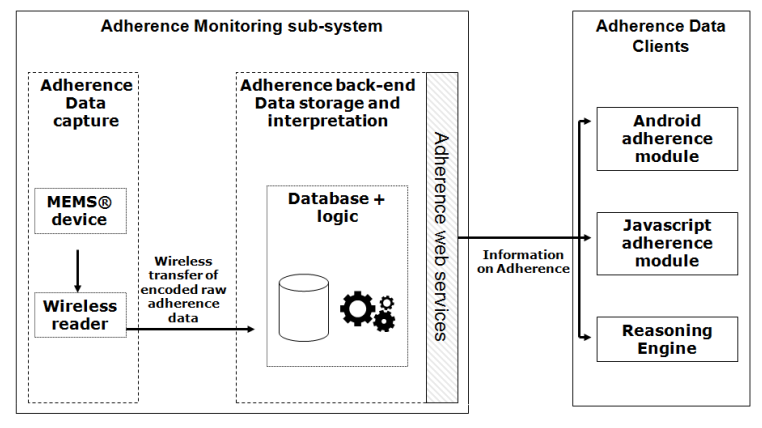

Figure 2. Adherence monitoring.

Data recorded by the MEMS ${ }^{\circledR}$ monitor are read by the wireless reader and sent to the adherence back-end for storage and interpretation. Only raw encoded adherence data are transmitted to the back-end. An algorithm is then used by the adherence back-end to reconstruct the dates and times of vial openings. Finally, these data are combined with contextual data such drug and regimen. The resulting information can then be used to provide information about patient's adherence and to support their interpretation.

Web-services give access to the resulting information on patients adherence. These web services can be access by different types of clients. An Android adherence module and Javascript adherence modules have been developed to facilitate the integration of the adherence data in Android apps and web applications. The reasoning engine embedded in the ICT4Depression system also relies on this webservice layer to retrieve inputs on adherence.

The communication between any client and the adherence web-service layer relies on REST/SOAP communication on secure HTTPS. In order to ensure a high level of security, a client certificate is required to access adherence web services. Additionally, a login restricts the access to the adherence data.

\subsubsection{Indirection Mechanism}

As mentioned in the previous section, the access to the adherence web-service layer requires a client certificate. The use of client certificates, especially in web browser, is often cumbersome for the end-user. A mechanism of indirection (Figure 3) has thus been implemented to avoid the storage of a client certificate on the end-user's machine without 
decreasing the security level required to access adherence data.

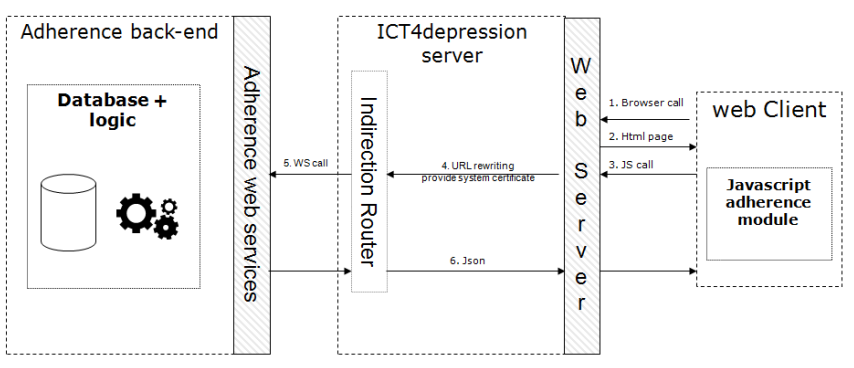

Figure 3. Indirection mechanism.

An indirection router acts as an intermediary between request from web clients and the adherence web-services response. This way, security constraints are delegated to the integrator.

\subsection{Reasoning Engine}

The aim of the Reasoning Engine is to offer personal guidance to a patient by means of continuous monitoring and feedback generation.

\subsubsection{Functional View}

Within the Reasoning Engines two core capabilities can be distinguished; on the one hand a rule-based approach is employed to assess the state of a patient. This is done by abstracting and interpreting specific aspects, such as physiological data acquired via the sensors, self-reports of mood, compliance to scheduled exercises and activities and the overall level of involvement with the system.

On the other hand a model-based approach is used to estimate the current influence and future developments of a therapy on a patient. By means of computational simulation of states associated with depression and therapeutic models [6], [2], [5], [4] (a so-called virtual patient [3]), a tailored feedback and therapeutic advice is provided to the patient. This advice is either meant to stimulate a patient and improve his involvement in the therapy, or to suggest the patient to perform a different type of therapy.

\subsubsection{Architectural View}

A number of steps are necessary to gather patient data, abstract it in regards of a temporal dimension, aggregate over different data sorts, reason about the current state and trends in patient's state and level of engagement with the system.

The Reasoning Engine architecture is made up of the following orthogonal components:

- Data Access Layer: provides a uniform interface to data sources (database and web services);
- Aggregation and temporal abstraction layer: implements the logic to discover trends in data and evaluate the influence of such trends on the human functioning.

- Rule-based reasoner: the workflow and rule management system Drools ${ }^{1}$ has been employed to react upon observed measurements and trends in physiological data, the level of engagement a patient has with the system as well as computational simulation outcomes. Reasoning about these aspects results in the generation of tailored feedback.

- Virtual Patient: implements computational models of states associated with depression and therapies as well as a parameter adaptation strategy to tailor simulation on the actual patient state. In terms of implementation a TherapyModel hierarchy of classes contains the business logic (computational models of depression) and a VirtualPatient class contains the constructor logic (parameter extraction, adaptation and simulation execution) used to provide tailored model-based support.

- Feedback notification: implements a message queue for dispatching feedback notifications generated by the Reasoning Engine. In order to avoid overloading the patient with these messages, a priority-based mechanism has been developed.

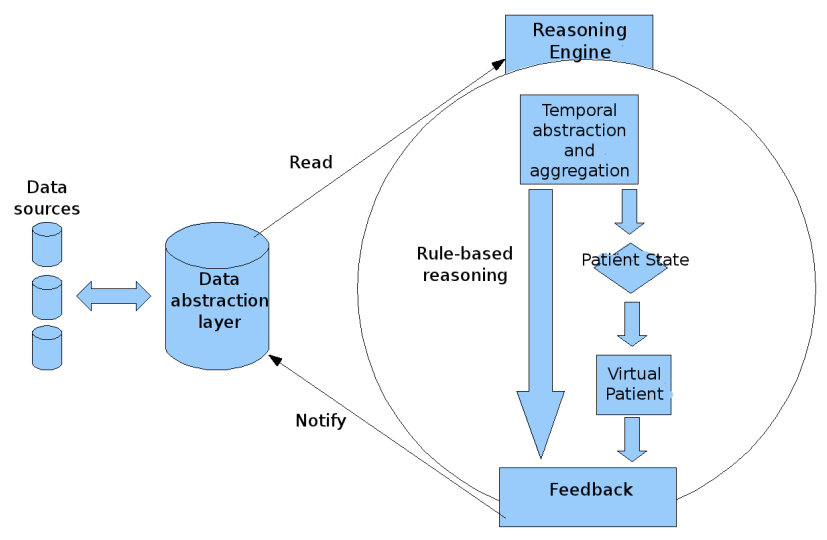

Figure 4. Reasoning Engine schematic architectural view.

A schematic representation of the Reasoning Engine architecture is depicted in Figure 4.

\subsection{Mobile Application}

The mobile application is several time addressed throughout the paper as one of the client tools for the

\footnotetext{
${ }^{1}$ http://www.jboss.org/drools
} 
therapeutic modules. This section focuses on describing the mobile application as a gateway of sensor data to the ICT4Depression back-end.

The software architecture chosen for sensor data collection and forwarding on the mobile phone is depicted in Figure 5 . The software functionality has been divided into four layers, each of which will be discussed in the following sections.

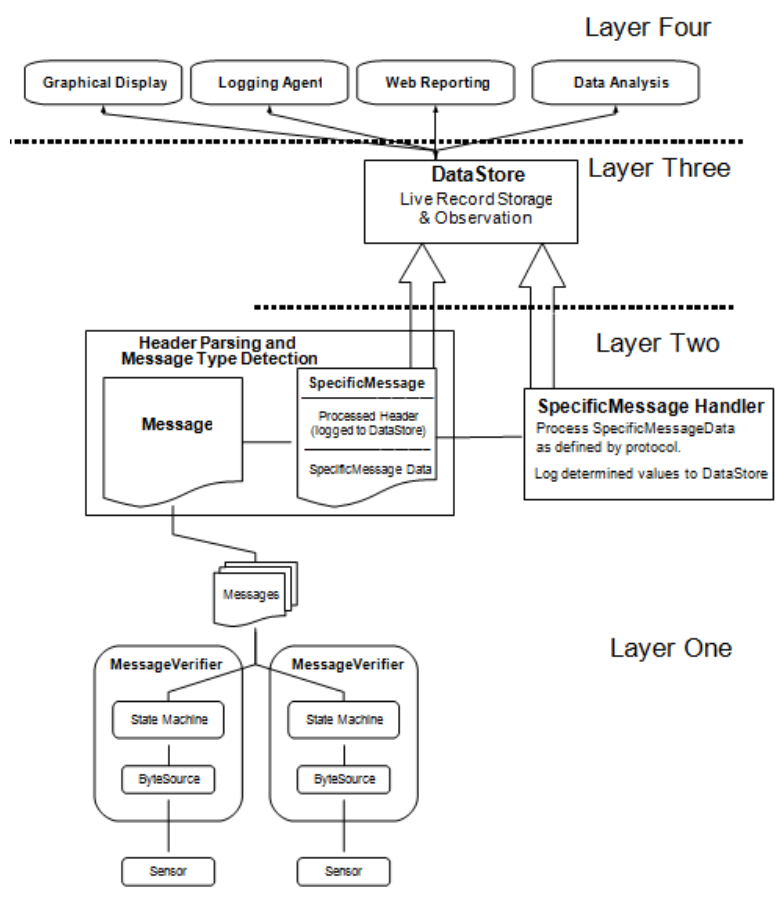

Figure 5. Mobile Sensor Management Software Architecture.

\subsubsection{Layer One Sensor Drivers}

Layer One is responsible for the collection of incoming messages through the Bluetooth radio. The latter provides incoming data in the form of byte streams. These byte streams are collected in layer One and assembled to generate full messages. A state machine is used to perform basic checks on the integrity of the messages received. Once a new message is received, the message is forward to Layer Two for further processing.

\subsubsection{Layer Two Message Assembly}

Upon receiving a message from Layer One, Layer Two is responsible for interpreting the message to the extent that the data contained in the message can be added to the data store in the appropriate way. Initially, the message header will be analyzed to determine how to further process the message. Information from the header is stored for future use by higher layers in the architecture. Based on the type of message, the payload is processed and stored in the data store.

\subsubsection{Layer Three The data store}

The data store contains entities called SensorRecords. These SensorRecords consist of values obtained from the sensors and a list of objects that have indicated to require a notification of any updates in these sensor values. If a SensorRecord is updated with a new value, these objects will be notified such that the architecture can promptly react to changing sensor readings.

\subsubsection{Layer Four The Agents}

The objects associated with the SensorRecords implement so-called agents. These agents act on new sensor data becoming available and can be responsible for tasks such as:

- Forward new data to the ICT4Depression Sensor Data Web Service (Web Reporting)

- Notifying the user, Raising alarms (Graphical Display)

- Processing and analyzing data stored on the mobile phone (Data Analysis)

- Changing values in SensorRecords (Logging Agent)

All interaction between the mobile phone and the higher layers in the ICT4Depression architecture makes use of such agents.

\subsection{Relapse Prevention}

Relapse rates are high after successful treatment for depression [1]. Prevention of relapses is therefore a major issue in the depression treatment.

In this project, among other techniques we are investigating the use of Relational Data Mining (RDM) to help physicians to identify conditions where relapse will most likely occur. We propose the use of Inductive Logic Programming (ILP) [13] as a RDM approach. We argue that ILP is highly adequate for the problem of predicting relapse situations.

ILP is a major field in Machine Learning with important applications in (Relational) Data Mining. The fundamental goal of an ILP system is to construct models for data. The main ingredients for ILP are: i) background knowledge; and ii) observations (usually called examples). It is usual to have two kinds of examples: positive examples (instances of the target concept) and negative examples (used to avoid overgeneralization). A characteristic of ILP is that both data and models are expressed in a subset of First Order Logic (FOL) providing and expressive language to encode both 
data and models. Background knowledge is a set of predicates encoding all the information that the domain expert finds relevant for constructing the models.

We can frame the relapse problem as a classification problem adequate for ILP. We have to provide the ILP system with the examples and the background knowledge. We define the target concept as a "situations that lead to relapse occurrences". Under this definition the positive examples are the set of situations where a patient undergoes a relapse episode. Negative examples are all other situations where the patient is "stable" or even improves. We distinguish two types of information to encode in the background knowledge: i) factual information; and analytical knowledge. The factual information consists in all the information that have been recorded and includes sensor's reading, answers to questionnaires and past medical records of the patient. The analytical knowledge allows temporal analysis of the factual information plus any another analysis that the experts thing may be useful to consider in the model.

\section{Conclusions}

An implementation of the service oriented architecture described in this paper has been implemented and deployed, including a client application for the web and another one for Android platform.

Most of the components of this architecture have been successfully tested in partial controlled trials. This is the case of the body-worn sensors and the mobile phone as gateway of sensory data.

The effectiveness of most of the therapeutic modules has been tested by the psychologists team in prior web interventions. However, a coherent domain model for these therapies has been put in place in the current implementation, which is accessed from two client applications developed by different partners. Furthermore, we have worked in close cooperation with the psychologists to design client applications which aim to enhance end-user experience, hopefully improving both adherence and outcome of the treatments.

The reasoning engine and its virtual patient model have also been tested and its outcomes are progressively being embedded in the clients application logic.

The medical adherence sub-system has been successfully integrated in the respective client applications. The recent implementation of an indirection mechanism is expected to further improve usability by reducing complexity for the end-user. Moreover, medical adherence sub-system is currently in use by other interventions world-wide.

Fully integrated end-user trials with real patients will be performed in the Netherlands and Sweden during the second half of 2012.

\section{References}

[1] C. Bockting, A. Schene, P. Spinhoven, M. Koeter, L. Wouters, J. Huyser, and J. Kamphuis. Preventing relapse/recurrence in recurrent depression with cognitive therapy: a randomized controlled trial. J Consult Clin Psychol, 73(4):647-57, 2005.

[2] F. Both, P. Cuijpers, M. Hoogendoorn, and M. C. A. Klein. Towards fully automated psychotherapy for adults - bas - behavioral activation scheduling via web and mobile phone. In A. L. N. Fred, J. Filipe, and H. Gamboa, editors, HEALTH$I N F$, pages 375-380. INSTICC Press, 2010.

[3] F. Both and M. Hoogendoorn. Utilization of a virtual patient model to enable tailored therapy for depressed patients. In ICONIP (3)'11, pages 700-710, 2011.

[4] F. Both, M. Hoogendoorn, M. Klein, and J. Treur. Modeling the dynamics of mood and depression. In Proceedings of the 2008 conference on ECAI 2008: 18th European Conference on Artificial Intelligence, pages 266-270, Amsterdam, The Netherlands, The Netherlands, 2008. IOS Press.

[5] F. Both, M. Hoogendoorn, M. C. A. Klein, and J. Treur. Computational modeling and analysis of the role of physical activity in mood regulation and depression. In Proceedings of the 17th international conference on Neural information processing: theory and algorithms - Vol. Part I, ICONIP'10, pages 270-281, Berlin, Heidelberg, 2010. Springer-Verlag.

[6] F. Both, M. Hoogendoorn, M. C. A. Klein, and J. Treur. Computational modeling and analysis of therapeutical interventions for depression. In Proceedings of the $2010 \mathrm{in}$ ternational conference on Brain informatics, BI'10, pages 274-287, Berlin, Heidelberg, 2010. Springer-Verlag.

[7] P. Cuijpers, J. Dekker, S. Hollon, and G. Andersson. Adding psychotherapy to pharmacotherapy in the treatment of depressive disorders in adults: a meta-analysis. J Clin Psychiatry, 70(9):1219-1229, 2009.

[8] P. Cuijpers, A. van Straten, S. Hollon, and G. Andersson. The contribution of active medication to combined treatments of psychotherapy and pharmacotherapy for adult depression: a meta-analysis. Acta Psychiatr Scand, 2009.

[9] P. Cuijpers, A. van Straten, A. van Schaik, and G. Andersson. Psychological treatment of depression in primary care: a meta-analysis. Br J Gen Pract, 59(559):51-60, 2009.

[10] ICT4Depression Consortium. Deliverable 5.2: Approved study protocol, 2011.

[11] C. Mathers and D. Loncar. Projections of global mortality and burden of disease from 2002 to 2030. PLoS Med, 3(11):e442, 2006.

[12] C. Melfi, A. Chawla, T. Croghan, M. Hanna, S. Kennedy, and K. Sredl. The effects of adherence to antidepressant treatment guidelines on relapse and recurrence of depression. Arch Gen Psychiatry, 55(12):1128-32, 1998.

[13] S. Muggleton and L. D. Raedt. Inductive logic programming: Theory and methods. Journal of Logic Programming, 19,20:629-679, 1994.

[14] N. Sood, M. Treglia, R. Obenchain, B. Dulisse, C. Melfi, and T. Croghan. Determinants of antidepressant treatment outcome. Am J Manag Care, 6(12):1327-36, 2000. 